\title{
Frontières
}

\section{Sagesse, mort et enseignement}

\section{Denis Jeffrey}

Volume 13, numéro 1, automne 2000

\section{La mort au tableau noir}

URI : https://id.erudit.org/iderudit/1074250ar

DOI : https://doi.org/10.7202/1074250ar

Aller au sommaire du numéro

\section{Éditeur(s)}

Université du Québec à Montréal

ISSN

1180-3479 (imprimé)

1916-0976 (numérique)

Découvrir la revue

Citer ce document

Jeffrey, D. (2000). Sagesse, mort et enseignement. Frontières, 13(1), 57-61. https://doi.org/10.7202/1074250ar

\section{Résumé de l'article}

Il y a de nombreuses questions sur la mort et la souffrance qui ne sont pas faciles à discuter dans la classe. À quel moment aborder ces questions dans le cheminement scolaire ? Comment s'y prendre ? À l'aide de quelles activités pédagogiques pouvons-nous sensibiliser des jeunes élèves à la souffrance, à la vieillesse, à la solitude, au suicide, au deuil ? Comment l'enseignant peut-il aborder en classe ces questions aussi délicates qu'essentielles ? Comment se servir du vécu des élèves pour les initier au thème de la mort ? On sait que ce thème délicat, dans le système scolaire, n'a jamais fait l'objet d'un enseignement rigoureux. Dans cet article nous nous demandons premièrement si la mort peut être l'objet d'un enseignement. Par la suite, nous proposons quelques courts textes de réflexion sur le thème de la mort et les enfants.
Ce document est protégé par la loi sur le droit d'auteur. L'utilisation des services d'Érudit (y compris la reproduction) est assujettie à sa politique d'utilisation que vous pouvez consulter en ligne.

https://apropos.erudit.org/fr/usagers/politique-dutilisation/ 


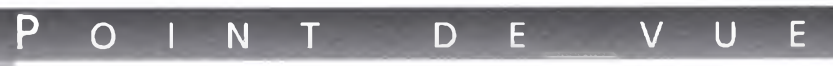

\section{Résumé}

II y a de nombreuses questions sur la mort et la souffrance qui ne sont pas faciles à discuter dans la classe. À quel moment aborder ces questions dans le cheminement scolaire ? Comment s'y prendre ? À l'aide de quelles activités pédagogiques pouvons-nous sensibiliser des jeunes élèves à la souffrance, à la vieillesse, à la solitude, au suicide, au deuil ? Comment l'enseignant peut-il aborder en classe ces questions aussi délicates qu'essentielles ? Comment se servir du vécu des élèves pour les initier au thème de la mort ? On sait que ce thème délicat, dans le système scolaire, n'a jamais fait l'objet d'un enseignement rigoureux. Dans cet article nous nous demandons premièrement si la mort peut être l'objet d'un enseignement. Par la suite, nous proposons quelques courts textes de réflexion sur le thème de la mort et les enfants.

Mots clés : mort-deuil - école - sagesse

\section{Abstract}

There are many questions on death and suffering that are not easy to discuss in the classroom. At what point should this subject be introduced? How should it be done? What pedagogical activities can we use to sensitize pupils about the subject of suffering, ageing, loneliness, suicide or mourning ? How can the teacher approach such questions that are so delicate yet so essential ? How can the lives of students be used to illustrate and initiate the theme of death ? We know that this delicate theme has never been the object of rigorous teaching in the scholastic system. In this article we ask whether death can be the object of teaching. By following this same thought, we propose some short texts to reflect on the theme of death among children.

Key words : death-mourning - schoolwisdom

\title{
Sagesse, mort et enseignement
}

\begin{abstract}
LE PETIT, UN VRAI KID (SIGNIFIE D'APRĖS MON HARRAP'S GAMIN, GOSSE) UN VRAI BOUT D'HOMME, JUSTE AU TOURNANT, JUSTE ET JUSTE. LA MOTO CHARGÉE DE NOTRE PROTECTION CIRCULAIT DEVANT, N'A PAS PU STOPPER NET AU SIGNAL DU BOUT D'HOMME. LES GARS QUI ÉTAIENT SUR LA MOTO AVAIENT CRU QUE C'ÉTAIENT DES COUPEURS DE ROUTE. ILS ONT TIRÉ. ET VOILÀ LE GOSSE, L'ENFANT-SOLDAT FAUCHÉ, COUCHÉ, MORT, COMPLÈTEMENT MORT. WALAHÉ ! FAFORO !
\end{abstract}

- Ahmadou Kourama, Allah n'est pas obligé

\begin{abstract}
Denis Jeffrey,
professeur au Département d'études sur l'enseignement et l'apprentissage, Faculté des sciences de l'éducation de I'Université Laval.
\end{abstract}

On n'aime pas vivre avec l'idée qu'on va mourir un jour, ne serait-ce que dans cent ans! Or, doit-on penser, à l'instar de Socrate, qu'il importe de se préparer à bien mourir ? Dans ses Essais, Montaigne souscrit à cette idée de Socrate selon laquelle philosopher, c'est apprendre à mourir. Cette vérité fulgurante fonde la vie philosophique et indique un chemin de sagesse. Les amoureux de la sagesse devraient, dans cette optique, consentir à côtoyer "philosophiquement » la mort. Comment interpréter, pour aujourd'hui, le sens de cette pratique philosophique ? Peut-on souscrire à cette conception de la sagesse ? Que signifie précisément apprendre à mourir ? Cette manière de philosopher peut-elle être empruntée par un jeune élève du niveau primaire?

\section{LA MORT À L'ÉCOLE}

Dans une classe de $2^{\mathrm{e}}$ année du primaire, un élève s'interroge librement sur le sens de la souffrance et de la solitude. Un enseignant " averti » y voit l'occasion de discuter un thème très difficile. Il prend le temps de proposer des lectures, de témoigner de ses expériences personnelles, de présenter quelques grandes idées sur le sujet, d'inciter l'élève à en parler avec ses parents et ses amis. Il y a de nombreuses questions existentielles qui ne sont pas faciles à considérer, ni en classe ni à la maison. À quel moment aborder ces questions dans le cheminement scolaire ? Comment s'y prendre ? À l'aide de quelles activités pédagogiques pouvons-nous sensibiliser des jeunes élèves à la souffrance, à la vieillesse, à la solitude, au suicide, au deuil ? Comment l'enseignant peut-il aborder en classe ces questions aussi délicates qu'essentielles ? Comment se servir du vécu des élèves pour les initier au thème de la mort? 
On sait que ce thème délicat, dans le système scolaire, n'a jamais fait l'objet d'un enseignement rigoureux. Ou bien l'enseignant ne sait pas comment l'aborder, ou bien on pense qu'il revient aux enseignants de religion ou à quelques autres spécialistes de le faire.

Demandons-nous si la mort peut être l'objet d'un enseignement. Le thème, en effet, ne s'impose pas de lui-même. Doiton parler de la mort et du deuil dans la classe uniquement lorsque survient un événement qui appelle une telle discussion ? Devons-nous plutôt élaborer une didactique de la mort et du deuil ou bien aborder ce thème d'une manière transversale, quand l'occasion se présente au cours d'une activité scolaire?

\section{LA SAGESSE DE SOCRATE}

$\mathrm{Si}$ on accepte, dans le sens de Socrate, que la sagesse s'acquiert en apprenant à mourir, demandons-nous de quel ordre est cet apprentissage ? Pour Socrate, côtoyer philosophiquement la mort signifie d'abord lever l'interdit dont elle est l'objet. La mort est interdite comme il est interdit de parler d'une corde dans une maison de pendu. Lever l'interdit sur la mort permet d'en discuter librement, de la prendre comme thème de méditation. Socrate, à vrai dire, pose la pierre angulaire d'une éducation à la mort en affirmant qu'elle peut être objet de réflexion. L'apprentissage de la mort consiste premièrement à libérer la parole à son égard

Dès lors, il n'est pas inutile de se demander si cet " apprentissage " doit constituer, dans la classe, un objet d'enseignement formel ou un objet d'enseignement informel. Un enseignement formel utilise des savoirs didactiques sur la mort pour l'enseigner dans le cadre d'activités précises. La mort devient alors une matière obligatoire dans le curriculum de l'élève, au même titre que la géographie et l'histoire. Dans le cadre d'un enseignement informel, la mort demeure un objet d'enseignement transversal enseigné par le truchement des autres activités d'apprentissage. À cet égard, il reviendrait à chaque enseignant d'accueillir les questions des élèves sur ce thème, ou de profiter d'une situation d'apprentissage - la lecture d'un conte où il est question de mort - pour proposer une discussion et donner quelques contenus d'apprentissage. Il est à souligner que l'enseignant n'a pas à attendre un événement tragique, tel le décès d'un élève, pour discuter de la mort et du deuil dans sa classe.

Pour ma part, je crois qu'il est préférable que la mort soit l'objet d'un ensei- gnement informel. Toutefois, je souhaite que chaque enseignant y soit bien préparé lors de ses études universitaires. Cette position me permet d'endosser la philosophie de Socrate. L'éducation à la mort ne formerait pas un programme comme tel, mais dépendrait du désir d'un enseignant de l'assumer. À cet égard, chaque enseignant pourra prendre en compte la maturité des élèves sur ces questions afin de respecter le fait que des élèves peuvent refuser de s'ouvrir à ces questions, et de respecter les croyances et les valeurs de la famille des élèves. Aussi, l'enseignant pourra respecter ses propres capacités d'aborder ce thème dans une classe.

On n'aborde pas avec les mêmes méthodes pédagogiques un cours sur la grammaire et un cours sur la mort. C'est que la grammaire constitue un savoir établi alors que la mort constitue à la fois un savoir établi (les rites funéraires, les statistiques sur le suicide, le concept de mort, etc.) et un savoir initiatique du fait que notre propre mort ne peut faire l'objet d'un savoir.

L'enseignement d'un savoir initiatique vise à susciter la réflexion, la compréhension et le questionnement sur soi. En d'autres termes, cet enseignement concerne la dimension existentielle, et implique des activités pédagogiques qui ouvrent sur une expérience personnelle, sur une mise en question de soi-même, sur une discipline personnelle (patience, concentration, méditation, réceptivité, sensibilité, souplesse et ouverture d'esprit, distance à soi, etc.), sur l'apprentissage de rituels de pacification (pour les émotions et sentiments excessifs de colère, de vengeance, de jalousie, de peur, d'impuissance, etc.), sur l'assomption de ses limites personnelles, sur la connaissance de son seuil de tolérance, sur l'acquisition d'un mode de vie permettant de trouver la paix intérieure, le sens à la vie, la sagesse.

La dimension existentielle renvoie, en somme, à un travail intime sur soi. C'est un travail dont les aspects affectifs et émotifs priment sur les aspects logiques et conceptuels. On peut supposer, en somme, qu'un enseignement informel sur la mort consiste à encourager un élève à faire un travail sur luimême : travail de réflexion, travail de compréhension, travail de concentration, travail de patience. Le chemin de la sagesse, dans le sens socratique, n'est-il pas celui de la quête de paroles pour dire l'indicible?

D'un point de vue pédagogique, la qualité d'une éducation à la mort dépend de l'intérêt de l'enseignant.
J'aimerais, pour terminer mon propos, présenter des situations de réflexion qui montrent l'importance du thème de la mort dans l'éducation des enfants.

\section{VIVRE SA MORT}

Nos parents nous ont vu naître, nos enfants nous verront mourir. On ne vit pas l'événement de sa mort, on la donne à vivre à ses proches. J'ai connu une femme, grand-mère d'un très bon ami, qui ne se sentait pas prête pour mourir. Bonne catholique, elle avait élevé une grande famille. Or, elle était hospitalisée depuis plusieurs années. Chaque jour, chaque semaine, chaque mois, on pensait qu'elle allait se décider à quitter la Terre. Il n'y avait rien à faire, elle ne voulait pas mourir. Elle avait peur de mourir. Elle ne voulait pourtant pas vivre, mais elle était incapable de mourir. La nuit, elle tremblait d'inquiétude. Le jour, elle ne pouvait ni manger ni boire. Elle avait peine à ouvrir les yeux. On l'entendait balbutier "Je ne veux pas mourir, je ne suis pas prête ". Elle n'était pas jeune cette grand-mère. Elle avait même eu la chance de connaître de nombreux arrière-petits-enfants. Elle souffrait. Sa vie intérieure n'était que souffrance, et elle glaçait de sa souffrance tous ceux qui venaient la voir. Un soir elle s'est évanouie à jamais dans le néant, seule, sans paroles d'au revoir. Tous les membres de la famille furent désemparés. Ils auraient tellement aimé que leur grand-mère traverse cette épreuve dans la sérénité. Une bonne préparation à la mort peut donner le courage de vivre, de vivre jusqu'au bout.

On lègue à ses proches et à ses amis sa manière de vivre et de mourir. C'est un cadeau extraordinaire. Les enfants ne sauraient recevoir plus beau cadeau que celui d'une vie et d'une mort courageuse, souriante et fraternelle.

La majorité, d'entre nous allons mourir à l'hôpital. Les enfants viennent à l'hôpital avec leurs parents. Ça sent bizarre l'hôpital. Des personnes en " jaquette " ouverte sur le dos et les fesses se promènent dans les longs corridors dont les planchers cirés invitent au patinage. La chambre est petite. Il y a une télévision placée en haut, sur le mur, devant le lit. Des fleurs habillent le chiffonnier en métal gris sur lequel on a déposé un pot à eau juste à côté d'un grand panier rouge rempli de chocolats et de petits pots de confitures. La chambre ne compte que deux chaises et un $L a-z-B o y^{\top M}$ vert, en cuirette. Un enfant doit grimper sur le lit pour 


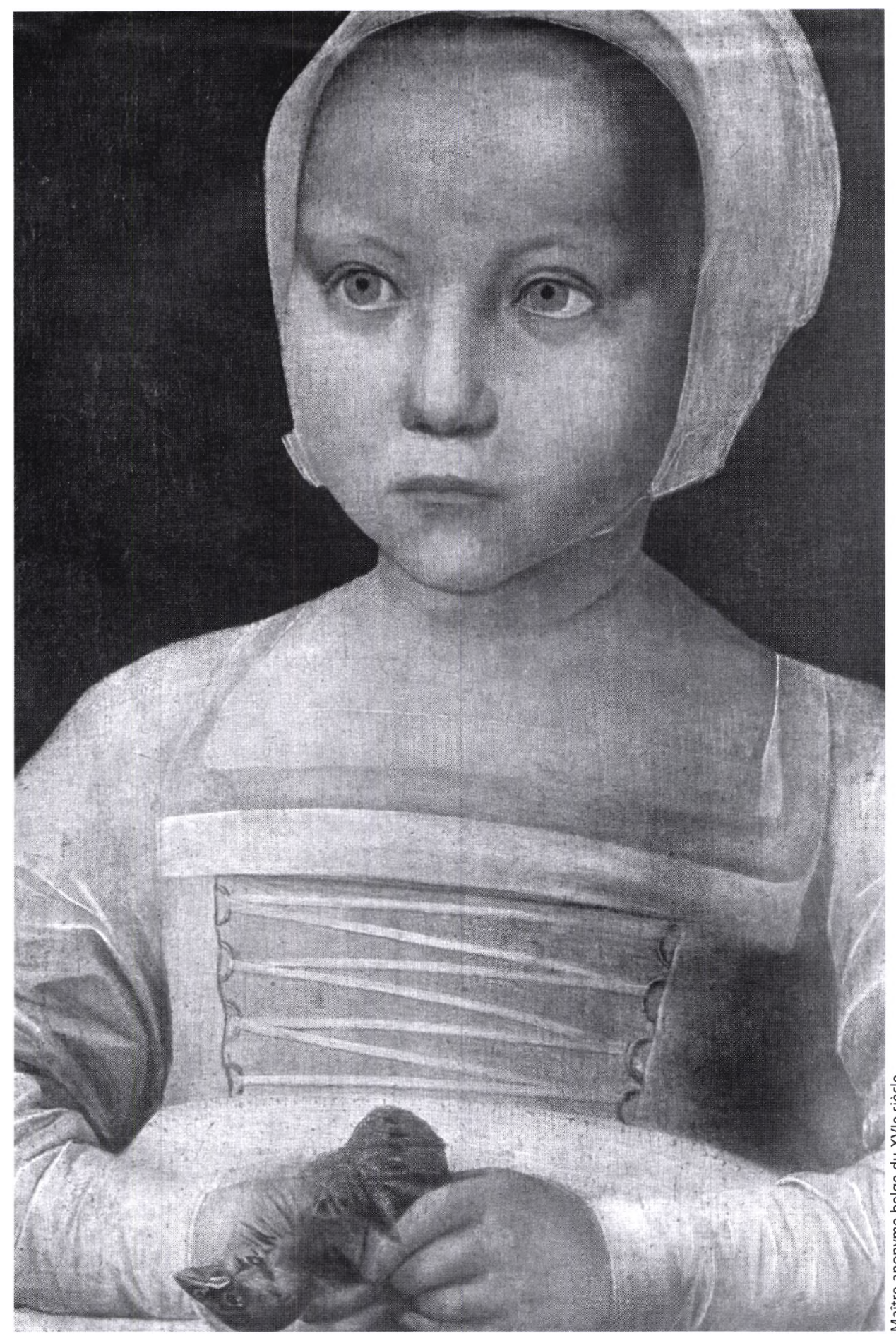

embrasser le malade. C'est difficile pour monter, car il y a des tubes et des bouteilles soutenues par un support métallique. L'enfant sait-il que dans cette aile de l'hôpital, on meurt tous les jours ? Sait-il que le malade mourra bientôt ? Peut-être voit-il pour la dernière fois son grand-père ou sa grand-mère, sa tante préférée ou son oncle qui voyageait tant. Comment lui dire la mort sans l'effrayer?

Nous naissons à l'hôpital et nous y mourrons. Nous n'avons pas vécu notre ment, parce que ça demande des heures d'effort. Je ne doute pas que beaucoup de personnes s'adonnent laborieusement à cette préparation. Pour les autres, il faudrait les aviser. Encore mieux, plus jeune on commence à se préparer à mourir en paix, plus facilement on affronte les difficultés de la vie. On devrait assurément apprendre à quitter la vie dès les premières années d'école.

Mourir soudainement, dit-on, fait partie des souhaits les plus répandus. Ils ne veulent pas voir la mort venir, ils ne veulent pas prendre le temps de mourir, prendre le temps de faire les derniers adieux, d'embrasser tout le monde, de prendre un dernier verre de vin et un dernier repas avant de disparaître à jamais. Ce sont de véritables modernes, si pressés. Ils n'ont pas pris le temps d'apprécier leur vie, lorsque leur heure sera venue, ils vont se précipiter tête première dans la mort. Ils ne prendront pas le temps de savourer cette toute dernière expérience de la vie. Cet empressement est assurément l'une des premières causes d'anxiété dans notre pays moderne.

\section{DU SALON FUNÉRAIRE À LA TOMBE}

La mort est quotidienne, mais son occultation l'efface de notre expérience. Nombre de jeunes garçons et de jeunes filles n'ont jamais vu un cadavre. Cela paraît normal puisque le rite d'exposition du défunt tend à disparaître. Pourquoi voir un cadavre ? Pourquoi des personnes veulent y toucher ? Accepte-t-on mieux le fait de la mort, de notre propre mortalité, si on a frôlé des doigts un corps sans vie ? Chacun pourra réfléchir à cela. Toutefois, mon expérience me porte à croire que la présence du cadavre, au salon funéraire, oblige à un respect du lieu et à un silence religieux. Aussi, ceux qui n'ont pas eu l'occasion de voir le défunt avant sa mort pourront lui tenir la main une dernière fois. Ça fait du bien de tenir la main de ceux qu'on a aimés, même jusque dans la mort. Il faut dire aussi que la présence du corps crée une atmosphère de recueillement et de fraternité. intensément les moments qui précèdent notre mort. C'est pourquoi on s'y prépa-
re. On prend soin d'être en paix avec soi et avec toutes nos relations. Je ne voudrais pas qu'une personne puisse dire qu'elle m'a haï toute sa vie. Ça me ferait mal de le savoir. Je suis par contre à l'aise avec l'idée que certains n'aiment pas mes idées, mon caractère ou ma personnalité. C'est même un peu normal de ne pas toujours être aimé pour ses idées. Pour mourir en paix, on doit peut-être, aussi, vivre en paix. On a toute la vie pour se préparer à mourir, heureuse-
Un enfant se présente avec ses parents au salon funéraire, salue ses cousins et cousines, attend dans la file pour exprimer ses condoléances. C'est la première fois qu'il vient au salon funéraire. Il est un peu gêné devant tout ce monde agité. Certains pleurent, d'autres s'embrassent, il y a même ces oncles, en retrait de la salle, qui fument des cigarettes en se racontant des blagues. 
On dirait que la tristesse se fait entendre lorsqu'on est près de la dépouille; en s'en éloignant, les rires et les conversations bruyantes prennent le dessus. L'enfant demande à ses parents ce qu'il doit dire à cet oncle qui a perdu sa conjointe. Il est embêté, il ne connaît pas les mots qu'on doit utiliser. Ses parents comprennent son embarras. Ils le rassurent, lui glissent à l'oreille les mots qu'il peut exprimer, ce qu'il faut faire, comment il faut se tenir. Pourra-til toucher le cadavre, demande-t-il ingénument ? Oui, bien sûr, lui répond-on, on peut toucher le cadavre. Tu peux lui caresser le front, lui prendre les mains. Une vieille tante a demandé le silence. Elle débite des prières sur un rythme très rapide, les autres répondent en chœur. Lorsqu'elle cesse de prier, chacun se retire dans son intimité pour penser à cette jeune parente décédée d'un accident de la route. On se prépare à quitter le salon funéraire pour l'office qui aura lieu au cimetière.

Heureusement qu'on est l'été. On a fait un grand trou dans le sol pour déposer le cercueil. Ça fait drôle de penser qu'on enterre une personne. Il faut s'assurer qu'elle est bien morte. Nul n'aimerait être enterré vivant. Les parents et les amis s'approchent du cercueil qu'on descend. Chacun prend une poignée de terre qu'il jette au fond du trou en disant : "Je vais toujours penser à toi ". L'enfant prend une poignée de terre et il demande à son père s'il doit prononcer les mêmes paroles. Il lui fait signe que oui. L'enfant s'approche du trou, une main lui agrippe l'épaule : c'est sa mère, elle l'a regardé avec des yeux qui disaient "Ne t'approche pas trop ". L'enfant a jeté sa poignée de terre en balbutiant : "Je vais essayer de ne pas t'oublier, tante Claire ». Ça lui a sorti sans y penser. Il voulait dire l'autre phrase, mais c'est celle-là qui est venue dans sa bouche. On lui a dit que c'était bien. Tout le monde est allé au restaurant, tout le monde semblait apaisé.

On ne vient plus souvent au cimetière pour visiter nos parents et nos amis décédés. Un jour, on ne se souvient plus du chemin pour trouver la pierre tombale. On finit par croire qu'on va se perdre, on ne veut plus y aller.

\section{L'ÉCOLE DE LA MORT ET LA MORT DANS L'ÉCOLE}

À l'école, l'automne dernier, l'enfant a participé à un projet sur la paix dans le monde. Avec son équipe, il a parlé à des enfants-soldats en Afrique. Il a présenté un documentaire déniché sur Internet. C'est l'histoire d'un enfant-soldat du

LA SAGESSE, C'EST D'ABORD UN ART DE LA MÉMOIRE

AFIN QU'ON NE RÉPĖTE PAS TROP SOUVENT

LES MÊMES VIOLENCES.

Sierra Leone qui raconte la guerre, la faim et la misère. Tous les membres de sa famille ont été tués avec des armes rudimentaires. On les a enterrés dans une fosse commune. Il n'y a pas eu de prière parce que durant la guerre, c'est trop dangereux. Il a une photo de Myriam, notre enfant-soldat. C'est une fille, mais c'est un soldat. Elle sait se défendre comme les garçons même si son uniforme est trop grand pour elle. Elle a un fusil et un casque militaire. Nous lui avons adressé des courriels en français, et elle a répondu. Elle nous a dit qu'elle vit dans la mort, mais qu'un jour, elle pourra manger à sa faim et vivre en paix. Elle est souvent triste parce que plusieurs de ses copains sont morts durant les batailles. Mais elle continue de croire à la vie, car elle a l'espoir.

Il n'est pas rare, sous nos cieux canadiens, de parvenir à la trentaine sans avoir jamais eu l'occasion de voir un mort "de près ». Expérience bien différente de celle que connaissent les habitants des pays, beaucoup trop nombreux sur la planète, où sévissent la famine, l'épidémie ou la guerre, ou les trois à la fois. Quel décalage entre nous et eux ! Là-bas, au loin, on vit dans la mort, mais est-on plus sage pour autant ? Est-on plus respectueux envers les morts et les vivants pour autant? Il n'y a pas lieu de le penser. Si on n'a pas la mémoire de ses morts, de ses guerres et de ses misères, comment peut-on être sage ? La sagesse, c'est d'abord un art de la mémoire afin qu'on ne répète pas trop souvent les mêmes violences. Ceux qui meurent, comment le dire, sont-ils morts pour rien ? Comment parler du sens de la mort avec un enfant? Doit-on lui parler de l'au-delà ? Quel sens prend la mort de tous ces hommes, ces femmes et ces enfants dans des pays tourmentés par la guerre ? Et ceux et celles qui ont souffert dans des salles de torture jusqu'à en perdre la raison, sont-ils morts pour rien?

Je me souviens des paysages de mon enfance, d'un monde beau, presque magique. Une famille bien agitée dans un quartier populaire où tous les jeunes mordaient dans la vie. Je mourrai avec ces souvenirs heureux. Au bout de la vie, lorsque nous mourrons, nous pensons à tous nos souvenirs heureux. Ça fait du bien, ça nous fait sourire. Notre bonheur n'est-il pas dans ces souvenirs que nous portons avec nous, et dans ces souvenirs heureux que nous fabriquons tous les jours ? Même durant la guerre, les hommes se fabriquent des instants de bonheur avec un geste d'affection, avec le chant d'un oiseau, avec l'odeur d'un aliment, avec un rêve de petite maison sise au creux d'une nature abondante.

Un enfant m'a dit que sa mère n'aime pas qu'il regarde la télévision quand il y a des scènes de violence. Il $\mathrm{y}$ a des morts, ça lui fait un peu peur mais pas trop peur, parce qu'il ne sait pas si c'est vrai. Un acteur meurt dans un film, mais il joue encore dans un autre film. Il fait semblant. Quelquefois cet enfant regarde des documentaires sur la guerre. Il sait que ce ne sont pas des acteurs. Les personnes meurent réellement. Sa mère pense que c'est préférable de regarder des documentaires éducatifs. Pourtant, dans les documentaires, la mort l'effraie parce que c'est la vraie mort.

Ce qui l'effraie encore plus, ce sont les grands dans la cour d'école. Ils viennent lui réclamer ses cartes de Hockey. Ils lui font peur parce qu'ils le menacent et qu'il est seul. Il n'en parle pas à sa mère ni à son père parce qu'ils ne peuvent pas venir le protéger. Il ne sait pas à qui en parler. Il pense que les grands lui font plus peur que les films avec des morts.

On dit qu'un enfant américain qui regarderait la télévision dès son retour de la classe verrait en moyenne une mort violente environ toutes les huit minutes. Comme le rappelle LouisVincent Thomas, "les hommes ont toujours aimé se repaître de la mort des autres $^{1}$ ». Certains considèrent qu'il n'y a rien de vraiment choquant dans le voyeurisme de la mort. Ce serait plutôt l'opposé qui serait choquant, c'est-à-dire si on occultait la mort. C'est vrai qu'à la télévision, on voit la mort de loin. Mais 
tout de même, il est préférable de la voir de loin que de ne plus la voir du tout. On voit de loin des personnes décédées dans des accidents d'avion, dans des tremblements de terre, des tornades, des ouragans, des déluges, des épidémies, des guerres, des actes de terrorisme. On voit de loin des gens mourir. Mais encore, ça nous rappelle la mort. Les images de la mort suscitent assurément moins de troubles que la réalité de la mort, mais nous préférons vivre la mort de loin.

\section{JOUER AVEC LA MORT}

Autrefois, mon frère s'est blessé en jouant avec des amis sur le toit de la maison. Un pommier facile à grimper, avec des grosses branches en escalier, conduit sur le toit. Mes parents ne voulaient pas qu'on monte sur le toit. C'est dangereux. Ça glisse et on peut tomber. Jean est quand même monté, pas tellement longtemps parce qu'il est tombé. Il a cassé sa jambe, son bras, son poignet et son majeur de la main droite. Il s'est aussi coupé à la tête et au visage. Une ambulance est venue pour le transporter à l'hôpital. Il s'est vraiment blessé parce qu'il ne peut pas tellement bouger. Il doit garder le lit. Mon oncle a dit qu'il aurait pu se tuer. Même s'il sait le danger, il aime bien jouer sur le toit.

Lorsque je revenais de l'école, j'avais des amis qui prenaient des risques pour se tuer. Ils se tenaient sur le pare-chocs arrière de l'autobus pour faire du skibottine. Ils pouvaient se tuer parce que l'autobus ou une voiture qui circulait derrière l'autobus pouvait les écraser. Ils savaient qu'ils pouvaient mourir ou se blesser gravement, mais ils le faisaient quand même. J'avais des amis qui prenaient des risques inutiles. Pour ma part, je faisais attention parce que je ne voulais pas faire de peine à mes parents. Ceux qui prennent des risques de cette sorte ne pensent pas à la peine qu'ils font à leurs frères, à leurs sœurs, à leurs parents, à leurs grands-parents et à leurs amis.

Les enfants jouent à la mort. Lorsqu'ils sont jeunes, ils jouent souvent à des jeux où l'on meurt : aux cow-boys et aux Indiens, aux soldats, à la cachette, à la police délivrance, à la traverse de Lévis, au ballon prisonnier. Tous ces jeux ont en commun de faire mourir les adversaires. Quand un joueur est mort, il sort du jeu. À la fin du jeu, celui ou celle qui n'est pas mort est le gagnant. Le jeu recommence indéfiniment, jusqu'à saturation. Quelquefois, lorsqu'ils jouent aux cow-boys, ils attachent les Indiens pour les torturer. Dans le jeu des enfants, il y a la mort et le retour à la vie. Ils meurent uniquement pour le temps du jeu.

Je ne serais pas triste si je meurs, mais je serais triste si une personne à laquelle je suis attaché meurt. Ce n'est pas que j'accorde plus d'importance à la vie des autres qu'à la mienne. C'est plutôt une question de destin. Par sagesse, j'ai toujours cru qu'on ne peut combattre le destin. On ne peut le tromper non plus. Ce n'est pas par passivité que j'accepte le destin, c'est que je crois que nous devons partir du destin pour aller plus loin. Le destin, on ne le change pas et on ne peut $y$ échapper, on ne peut qu'y ajouter son grain de sel. Je me souviens d'avoir lu que le mot "salaire » provient du mot "sel ». Avant l'ère moderne, le sel a été une monnaie d'échange. Ajouter du sel à son destin, c'est ce qu'il en coûte pour ne pas se rendre aveugle à ses actes, comme CEdipe qui tua son père et maria sa mère. Notre salaire est le produit de ce sel. Il faut être un peu cuisinier pour mourir heureux.

\section{PENSER SA MORT ET L'APRĖS-VIE}

Ma mort surviendra comme un dérèglement prévisible de mon organisme : l'infarctus ou le cancer. Les statistiques de santé créent notre destin. C'est pile ou face. Il y a encore quelque chance de mourir dans un accident routier. Il y a moins de chance de mourir dans un accident d'avion ou dans un accident de travail. Le sort est jeté, on peut s'attendre à mourir d'un infarctus ou d'un cancer. C'est une loi quasi canonique dans notre beau pays. Les enfants l'apprennent assez tôt, dans leur famille on meurt du cancer ou du cour. C'est comme ça, on n'y peut rien.

Mourir demeure un scandale difficile à accepter. On ne se résigne pas à disparaître sans laisser de traces. Dans Le Banquet, Platon soutient que tout désir est désir d'éternité. Selon leur personnalité et leur culture, les uns veulent s'éterniser dans leurs enfants, les autres en créant des objets d'art, d'autres résisteront au temps par leurs écrits et leurs idées. Il y en aura qui seront de véritables héros. On se souviendra d'eux pour l'éternité. Par son sexe, sa plume, son scalpel, son pinceau ou son marteau, chacun cherche à se rendre immortel. Ce désir d'éternité permet à l'humanité de cumuler des documents et des monuments qui forment sa mémoire. Si un jour les hommes disparaissent de la surface de la terre, parce que tous les hommes sont mortels, il restera au moins cette mémoire pour les extra-terrestres.

Je ne sais pas si la vie continue après la mort. Je n'arrive pas à y croire. Je n'ai pas de raison d'y croire. Serais-je une meilleure personne si j'y croyais ? Je ne le pense pas. Alors pourquoi y croire? Pour faire plaisir aux croyants ! Je préfère ouvrir toutes les portes de l'au-delà aux enfants. Chacun vivra ses propres expériences de la vie et de la mort.

\section{CONCLUSION}

Les souvenirs heureux nous sont chers. On y revient, parfois, pour se donner le courage d'affronter une situation tragique. Trop d'événements malheureux rappellent, en fait, que la vie est fragile. $\mathrm{Ne}$ dit-on pas que la vie s'apprécie à l'aune du sentiment de sa perte? Les riches réflexions sur la fragilité de la vie, sur le vieillissement que chacun redoute, sur le fait que la vie peut s'interrompre à tout moment, devraient-elles nous aider à mieux assumer notre destin de mortel ?

\section{Note}

1 Louis-Vincent THOMAS, La mort en question, Paris, L'Harmattan, Nouvelles Études Anthropologiques, 1991, p. 171. 